\title{
Luces y sombras en la implementación del Acuerdo de Tierras en Colombia*
}

\author{
María Rocío Bedoya Bedoya (Colombia)**
}

\section{Resumen}

El primer punto del Acuerdo de Paz entre el Gobierno colombiano y las Fuerzas Armadas Revolucionarias de Colombia (FARC) establece la necesidad de un nuevo modelo que revitalice la producción agrícola y que beneficie al pequeño campesino, comprometiendo al Gobierno en la configuración de un modelo de desarrollo rural integral que se ocupe de los problemas del campo; sin embargo, tanto el gobierno de Juan Manuel Santos como el del actual presidente, Iván Duque, le apuestan a un modelo neoliberal y extractivista que parece ir en contravía del Acuerdo de La Habana. A partir de la estrategia de investigación cualitativa documental, este artículo da cuenta de las convergencias y divergencias que se derivan del Acuerdo de Tierras y las medidas impulsadas por el Gobierno, con el fin de allanar el camino hacia la reforma rural integral. Como conclusión, se advierte que los decretos del Fast Track en materia rural van en contravía de la paz territorial, por cuanto se desconoce la autonomía de los territorios y se da prevalencia a las decisiones del Gobierno central.

\section{Palabras clave}

Proceso de Paz; Posconflicto; Territorio; Reforma Rural Integral; Colombia.

Fecha de recepción: febrero de 2018

- Fecha de aprobación: septiembre de 2018

\section{Cómo citar este artículo}

Bedoya Bedoya, María Rocío. (2019). Luces y sombras en la implementación del Acuerdo de Tierras en Colombia. Estudios Políticos (Universidad de Antioquia), 54, pp. 37-58. http://doi.org/10.17533/ udea.espo.n54a03

\footnotetext{
* Este artículo se deriva de la investigación Diagnóstico socioeconómico de las comunidades de Flor del Monte, La peña y Santa Rafael del municipio de Ovejas, departamento de Sucre, inscrita en el Comité para el Desarrollo de Investigación (CODI), Universidad de Antioquia, mediante acta N. ${ }^{\circ} 10$ del 17 de julio de 2014. Una versión de este artículo fue presentada en el XVI Congreso Internacional sobre Integración Regional, Fronteras y Globalización en el Continente Americano el 19 de octubre de 2017, Medellín, Antioquia.

** Abogada. Especialista en Derecho Laboral y de la Seguridad Social. Magíster en Ciencia Política. Magíster en Empleo, Relaciones Laborales y Diálogo Social. Doctora en Gobierno y Administración Pública. Grupo Derecho y sociedad, Facultad de Derecho y Ciencias Políticas, Universidad de Antioquia UdeA. Calle 70 No. 52-21, Medellín, Colombia. Correo electrónico: maria.bedoya@udea. edu.co
} 


\title{
Lights and Shadows in the implementation of the Land Reform Agreement in Colombia
}

\begin{abstract}
The first point of the Peace Agreement, between the Colombian Government and the Revolutionary Armed Forces of Colombia (FARC), establishes the need for a new model that revitalizes agricultural production and that benefits the small farmer, committing the Government in the configuration of a model of integral rural development that addresses the problems of the countryside; However, both, the government of Juan Manuel Santos and the current president, Iván Duque, are betting on a neoliberal and extractivist model that seems to go against the Havana Agreement. Based on the documentary qualitative research strategy, this article gives an account of the convergences and divergences that derive from the Land Agreement and the measures promoted by the Government, in order to pave the way towards comprehensive rural reform. In conclusion, Fast Track decrees in rural areas go against territorial peace, because they do not take the autonomy of the territories into account, and give prevalence to the decisions of the central government.
\end{abstract}

\section{Keywords}

Peace Process; Postconflict; Territory; Integral Rural Reform; Colombia. 


\section{Introducción}

El problema de la tierra contempla sus formas de propiedad y de distribución, como también las maneras de producción y usufructo; por lo cual, el modelo de desarrollo agrario y sus consecuencias en la sociedad han estado en el centro de los conflictos sociales y armados de Colombia durante toda su historia republicana. Particularmente, durante el último siglo la sucesión de movilizaciones campesinas, así como los ensayos de reforma agraria y la expropiación violenta por desplazamiento forzado han tejido la historia de la cuestión agraria.

En 1936, durante la República Liberal, el intento reformista de la Revolución en Marcha del presidente Alfonso López Pumarejo se plasmó en la reforma constitucional que afirmaba la función social de la propiedad y en la Ley 200 que contemplaba el derecho de los trabajadores rurales a la propiedad de la tierra, además de la extinción de dominio en un término de diez años para las tierras inexplotadas y creaba la jurisdicción agraria. Ese solo intento de adecuación del mercado de tierras a las necesidades de una economía capitalista en ascenso, originó la reacción de los sectores más conservadores ligados a la gran propiedad terrateniente y beneficiarios de unas relaciones de producción precapitalistas en el campo que mantenían a las masas de campesinos, indígenas y afrocolombianos atados a una dependencia servil y semifeudal de los grandes propietarios (Fajardo, 1984).

No solo fue el ensayo de levantamiento militar contra el segundo gobierno de López Pumarejo (1944), sino la presión desde el Parlamento para la expedición de la Ley 100 de 1944 que prolongaba los contratos de arrendamiento y aparcería, además, ampliaba de diez a quince años el plazo para la extinción de dominio de las tierras inadecuadamente explotadas en beneficio del Estado; también la violencia desatada contra los pobres del campo que, desde entonces, mediante las masacres y el desplazamiento forzado han producido sucesivas olas de despojo, y verdaderas contrarreformas agrarias con las que los grandes propietarios han respondido a los intentos de democratización del campo (Semillero de Estudios Políticos Rurales, 2010, 15 de diciembre).

Más adelante, en pleno Frente Nacional, con la Ley 135 de 1961 de «Reforma Social Agraria» y la Ley 1 de 1968, se crearon el Instituto Colombiano para la Reforma Agraria (Incora) y el Fondo Nacional Agrario para el impulso de la distribución de tierra a campesinos sin tierra y el apoyo para la adecuación y el riego para la producción de la pequeña propiedad. 
Igualmente, se creó por ley la Asociación Nacional de Usuarios Campesinos (ANUC) y se reglamentó la extinción de dominio por vía administrativa.

Sin embargo, la presión de las masas de campesinos por la tierra, frente a las expectativas no cumplidas de la reforma agraria, produjo a comienzos de la década de 1970 una gran movilización nacional de invasiones y tomas de tierra bajo la consigna: «La tierra para el que la trabaja». Esas luchas sociales fueron la réplica de los movimientos de reivindicación agraria que desde comienzos de siglo alentaron la organización de los trabajadores del campo (PNUD, 2011). A pesar de la represión y la Violencia de las décadas de 1940 y 1950 contra los pobres del campo, la agitación social seguía denunciando un problema no resuelto, fuente permanente de los conflictos agrarios. Es por ello que, desde su nacimiento en 1964, la guerrilla de las Fuerzas Armadas Revolucionarias de Colombia (FARC) presentó su Programa Agrario como base fundacional y política de su movimiento social y armado (Villamizar, 2017).

Al igual que en la década de 1940, la reacción de los grandes latifundistas y grandes propietarios agrarios combinó la violencia con la contrarreforma legal. El Pacto de Chicoral entre el Gobierno nacional y los grandes propietarios

[ 40 ] de la tierra, congeló cualquier proyecto de reforma agraria redistributiva y redujo el papel del Incora y de las instituciones de política pública agraria a estímulos de la gran producción agropecuaria mediante las Leyes 4 de 1973 y 6 de 1975 (Palacios, 1995, p. 257).

Más adelante, en la Constitución de 1991 en su artículo 58 se hace un resumen de los avances normativos al afirmar que: «se garantizan la propiedad privada y los demás derechos adquiridos con arreglo a las leyes civiles, los cuales no pueden ser desconocidos ni vulnerados por leyes posteriores»; sin embargo, «cuando de la aplicación de una ley expedida por motivo de utilidad pública o interés social, resultaren en conflicto los derechos de los particulares con la necesidad por ella reconocida, el interés privado deberá ceder al interés público o social». Igualmente recoge y actualiza el sentido de la reforma constitucional de 1936: «La propiedad es una función social que implica obligaciones. Como tal, le es inherente una función ecológica». El artículo 64 avanza en reconocer el deber del Estado de:

Promover el acceso progresivo a la propiedad de la tierra de los trabajadores agrarios, en forma individual o asociativa, y a los servicios de educación, salud, vivienda, seguridad social, recreación, crédito, 
comunicaciones, comercialización de los productos, asistencia técnica y empresarial, con el fin de mejorar el ingreso y calidad de vida de los campesinos (Asamblea Nacional Constituyente, 1991).

Finalmente, señala que «El Estado protegerá y promoverá las formas asociativas y solidarias de propiedad» (artículo 58).

Posteriormente, en 1994 la Ley 160 estableció un mercado subsidiado de tierras para el acceso a la propiedad de los pequeños campesinos, estableció un límite a su extensión (UAF) y creó las zonas de reserva campesina para proteger la economía familiar de la extensión del latifundismo agrario.

En efecto, durante las décadas de 1970, 1980 y 1990 la aparición del narcotráfico y el paramilitarismo, al igual que su relación con sectores de la gran propiedad agraria y de la misma clase política tradicional, estimularon una nueva oleada de contrarreforma agraria mediante la violencia. Las dimensiones de la crisis de derechos humanos y de la degradación que la guerra ha producido sobre la dignidad de las personas y sobre la calidad moral de la vida colombiana están ampliamente recogidas en el informe del Centro Nacional de Memoria Histórica (2013), iBasta ya! Colombia: memorias de guerra y dignidad.

El propósito de este artículo es examinar el proceso de implementación del Acuerdo de Paz en torno a lo que las partes denominaron desarrollo rural integral. En la primera parte se exponen los puntos centrales del acuerdo inicial de La Habana; en la segunda, se analizan algunas de las medidas de implementación del Acuerdo sobre desarrollo rural integral referidas a los decretos del Fast Track, asimismo, revisa la Ley Zidres de 2016 y las recomendaciones de la Misión Rural de 2015; finalmente, se destacan los aspectos positivos y negativos de este proceso de implementación.

Se revisaron las diversas fuentes relacionadas con el tema de las tierras, incluyendo el primer punto del texto del Acuerdo Final; además, se revisó el contenido de los decretos 902, 896, 893, 892, 884, 890 y 882 de 2017 y otras fuentes documentales como la Ley Zidres (1776 de 2016), su decreto reglamentario y la Misión Rural, cuyos contenidos están encaminados a implementar la reforma rural integral.

En definitiva, con este artículo se espera hacer un balance del proceso de implementación que ha propuesto el Gobierno colombiano en materia 
del Acuerdo de Tierras hacia un nuevo campo colombiano, con miras hacia la construcción de una paz estable y duradera; asimismo, advertir sobre los riesgos y las posibilidades que este proceso supone.

\section{Negociaciones de paz}

Desde la década de 1980 se han venido produciendo múltiples esfuerzos de construcción de paz, tanto por parte de los actores en conflicto, como de la sociedad colombiana. Durante este periodo se registraron los esfuerzos de diferentes gobiernos en la búsqueda de la paz, entre los cuales se destacan los de Belisario Betancur, Virgilio Barco, César Gaviria, Ernesto Samper, Andrés Pastrana, Álvaro Uribe y Juan Manuel Santos; asimismo, se ha dado la desmovilización negociada de varios grupos armados ilegales desde 1990, tales como el Movimiento 19 de abril (M-19), el Ejército Popular de Liberación (EPL), el Partido Revolucionario de los Trabajadores (PRT), el Movimiento Armado Quintín Lame (MAQL), la Corriente de Renovación Socialista (CRS), las Milicias Populares de Medellín y las Autodefensas Unidas de Colombia (AUC). Durante el gobierno de Juan Manuel Santos se llegó a un acuerdo de fin del conflicto con las FARC-EP y actualmente se negocia con dificultades un acuerdo con el Ejército de Liberación Nacional (ELN).

Estas dos últimas negociaciones tienen su simiente en junio de 2010 cuando fue elegido presidente Juan Manuel Santos. Desde ese mismo año se iniciaron conversaciones discretas con las FARC y a mediados de 2012 se anunció públicamente un proceso de negociación que se instaló en Oslo con apoyo de la comunidad internacional, aunque la Mesa de Conversaciones estableció su sede permanente en La Habana, Cuba. En mayo de 2013 las partes hicieron público el Acuerdo sobre las Tierras (Villarraga, 2016).

Para este artículo se realizó un balance a partir de la exploración de los contenidos del Acuerdo Final, los cuales se contrastaron con las medidas de implementación adelantadas por el Gobierno, las reacciones de la Procuraduría General de la Nación y la Consultoría para los Derechos Humanos y el Desplazamiento (Codhes).

Si bien los acuerdos de paz de La Habana constituyen un avance significativo en la finalización del conflicto armado, como un punto de partida para la construcción de una paz sostenible, en materia de tierras el conflicto en el campo colombiano ha sido tan profundo que requiere cambios estructurales en la esfera social rural. Los efectos del conflicto han sido múltiples y su 
reparación y no repetición requieren de gran voluntad política, cambio de enfoques, nuevo modelo de desarrollo rural, nueva institucionalidad, mayor presupuesto y gran ánimo y decisión de reconciliación.

Estas cuestiones aún se ven muy lejanas, tanto por las medidas tímidas adoptadas por el gobierno Santos para su implementación, como por las tendencias del nuevo gobierno perteneciente al partido político Centro Democrático, el cual representa, en materia de desarrollo rural, todo lo contrario a lo estipulado en el Acuerdo de Paz.

\section{El Acuerdo de Tierras: el primer acuerdo de La Habana}

En junio de 2013 se dio a conocer a los colombianos que la Mesa de Conversaciones entre el Gobierno y las FARC había llegado a un acuerdo sobre el primer tema de la agenda: Desarrollo agrario integral con enfoque territorial. Muchos colombianos consideraron que este era un buen mensaje, ya que se estaba avanzando en la discusión y concreción de un acuerdo y se estaba mostrando la posibilidad de un arreglo en temas tan simbólicos, sensibles y controversiales como el agrario, lo cual significaría que sí es posible solucionar el conflicto armado conversando (Vargas, 2013).

Como se ha dicho, el primer punto de la negociación entre el Gobierno y las FARC fue el referido al desarrollo rural integral, aspecto de gran relevancia, dado que precisamente es el problema de concentración de la tierra en Colombia y las desigualdades que este ha generado lo que dio lugar al surgimiento de la guerrilla de las FARC (Villamizar, 2017). En materia de tierras, se acordó la constitución de un Fondo de Tierras para la Reforma Rural Integral, cuyo propósito es:

Lograr la democratización del acceso a la tierra en beneficio de los campesinos y de manera especial las campesinas sin tierra o con tierra insuficiente y de las comunidades rurales más afectadas por la miseria, el abandono y el conflicto, regularizando los derechos de propiedad y en consecuencia desconcentrando y promoviendo una distribución equitativa de la tierra (Gobierno y FARC-EP, 2017, p. 14).

Este Fondo de tres millones de hectáreas se surte de tierras provenientes de la extinción de dominio, baldíos recuperados a favor de la nación, tierras provenientes de la actualización de la Reserva Forestal, tierras adquiridas por el Estado para estos propósitos y tierras donadas para el Fondo de Tierras. 
Asimismo, se decidió diseñar un plan masivo de formalización de la pequeña y mediana propiedad rural, y formación y actualización del catastro e impuesto predial rural, debido a los altos niveles de informalidad que existen en Colombia en materia de titulación de tierras. También se acordó promocionar planes nacionales de desarrollo con enfoque territorial (Gobierno y FARC-EP, 2017, p. 16), lo cual supone un compromiso con las necesidades de cada territorio y las particularidades de sus habitantes (Bedoya, 2015).

El Acuerdo creó los Programas de Desarrollo con Enfoque Territorial (PDET) con el objetivo de «lograr la transformación estructural del campo y el ámbito rural, y un relacionamiento equitativo entre el campo y la ciudad» (Gobierno y FARC-EP, 2017, p. 47). Incluye criterios de priorización de territorios, planes de acción para la transformación regional y mecanismos de participación activa de las comunidades.

Con los planes nacionales para la reforma rural integral se asumió, además, la responsabilidad de construir la infraestructura necesaria para garantizar el desarrollo social y el estímulo a la productividad campesina. Asimismo, se debe adoptar una política de seguridad alimentaria y trabajar en función del fortalecimiento de los mercados regionales.

Si bien se registran avances importantes en esta materia, es preciso señalar que aún quedan asuntos pendientes de hondo calado en lo que se refiere a la reforma agraria: el latifundio, la delimitación de la propiedad, las políticas públicas que prohíban la extranjerización del territorio, la regulación sobre la extracción minero-energética y de agrocombustibles que respete la vocación agrícola de los territorios, la autonomía de la comunidades y las bases del modelo de desarrollo rural integral acordado en La Habana; del mismo modo, se precisa de una renegociación de los tratados de libre comercio, los planes de ordenamiento territorial, el uso del suelo y el derecho real de superficie, además de la creación del Consejo Nacional de la Tierra (Villarraga, 2016). Todo lo anterior indica que el gran pendiente de la negociación es el modelo económico.

\section{Medidas de implementación}

Con posterioridad a junio de 2013, fecha en la que se produjo el primer acuerdo de La Habana, el Gobierno nacional ha impulsado distintas medidas, algunas de ellas con la finalidad de implementar el Acuerdo con los decretos del Fast Track de 2017 y otras en contradicción de este, como la Ley Zidres o 
Ley de Baldíos (Ley 1776 de 2016). En 2015 el Gobierno encargó a la Misión para la Transformación del Campo Colombiano conocida como Misión Rural, un análisis profundo de las realidades del desarrollo rural en Colombia.

\subsection{Zonas de Interés de Desarrollo Rural, Económico y Social (Zidres)}

El 29 de diciembre de 2016 se aprobó la Ley 1776, por la cual se crean y se desarrollan las Zonas de Interés de Desarrollo Rural, Económico y Social (Zidres), con el propósito de regular el tema de baldíos que tiene enormes implicaciones para la construcción del proceso de paz en Colombia. Estas deben cumplir con los siguientes requisitos: a) que estén geográficamente aisladas de los centros urbanos más significativos; b) que demanden elevados costos de adaptación productiva; c) que tengan baja densidad poblacional y altos índices de pobreza; d) que carezcan de infraestructura mínima para el transporte y comercialización de los productos; y e) que se adecúen y correspondan a la internacionalización de la economía, sobre bases de alta competitividad, equidad, reciprocidad y conveniencia nacional.

El Gobierno nacional, mediante el Decreto 1273 del 3 de agosto de 2016, reglamentó la integración, organización y funcionamiento del Sistema Nacional de Desarrollo Rural para las Zidres a través de la coordinación del Ministerio de Agricultura y Desarrollo. Para ello, se crearon instrumentos que permitan el fomento de proyectos productivos, además de incentivos y estímulos para los proyectos productivos y garantías para los proyectos Zidres.

Por su parte, la Corte Constitucional, en sentencia C-077 de 2017, condicionó la exequibilidad de la Ley 1776 de 2016 a la información, concertación y coordinación con los Concejos Municipales de cada territorio. En consecuencia, el 7 de febrero de 2018 el Departamento Nacional de Planeación (DNP) expidió el documento Conpes 3917, el cual define las áreas de referencia como insumo para la identificación de las Zidres. Con la identificación de 7,2 millones de hectáreas como potenciales, el Gobierno avanzó hacia su implementación.

La Ley Zidres se propone como principal objetivo construir un nuevo modelo de desarrollo económico regional que parte de promover el acceso y la formalización de la propiedad de la tierra, la inclusión social y productiva a los campesinos, los trabajadores agrarios, las mujeres rurales, los jóvenes rurales y los ocupantes tradicionales de bienes de la nación. Se incluye: 
impulsar el desarrollo de infraestructura para la competitividad de las Zidres; procurar la responsabilidad social empresarial (RSE) y la responsabilidad ambiental empresarial (RAE) de las personas jurídicas que desarrollen proyectos en las Zidres; priorizar iniciativas destinadas a la producción de alimentos. Asimismo, se propone promover el desarrollo regional; promocionar y consolidar la paz y la convivencia; impulsar la realización de proyectos agrícolas y pecuarios; facilitar procesos asociativos empresariales; constituir zonas francas agroindustriales; crear parques de ciencia, tecnología e innovación y centros de investigación y desarrollo de la agricultura tropical y de investigación en biodiversidad y recursos naturales; construir una oferta científico tecnológica; construir modelos habitacionales rurales; desarrollar procesos de producción familiar y comunitaria; y procurar el manejo sostenible de los recursos naturales mediante procesos técnicos dirigidos por expertos (Ley 1776, 2016).

El propósito real de la Ley Zidres queda al descubierto al leer el conjunto de esta y su exposición de motivos. Estas zonas se crearon para «eliminar los límites a la propiedad» que han impedido que grandes empresas interesadas en la altillanura colombiana - mencionada en el proyecto de ley como una de las regiones aptas para la constitución de estas zonas-, concreten la compra de vastas extensiones de tierra para dar paso a sus proyectos agroindustriales, situación que venía ocurriendo antes de la aprobación de la ley; y adicionalmente, con el fin de favorecer a estos empresarios con «incentivos» para sus proyectos privados, mediante la financiación con recursos públicos (Castilla, 2015).

Por otro lado, la Ley presenta como beneficios para los campesinos, los trabajadores agrarios, las mujeres rurales y los jóvenes rurales algunos de los objetivos de las Zidres: acceso y formalización de la propiedad de la tierra, y la inclusión social y productiva. Sin embargo, el enfoque de la Ley desconoce los niveles de pobreza de la mayoría de los habitantes del campo y la responsabilidad del Estado en la situación de abandono del campesinado. No obstante, esta Ley facilita los procesos asociativos empresariales y la construcción de zonas francas agroindustriales, con lo cual favorece los grandes proyectos agroindustriales que muy seguramente convertirán al campesinado en un grupo de asalariados, en lugar de propietarios con real disposición sobre sus tierras (Ley 1776 de 2016).

Otro de los problemas que los opositores ven en la Zidres es que los baldíos de la nación podrían ser arrendados para la agroindustria — la cual 
tiene el capital para adecuar la tierra e incluso impulsar la construcción de infraestructura- y no para familias pobres sin tierra, como lo estableció la Ley 160 de 1994. Esto revela que el Gobierno propone una asociatividad en la desigualdad, esto es, el impulso de proyectos entre campesinos pobres y grandes empresas agrícolas, forestales y ganaderas, estas últimas con alta capacidad económica y en claras condiciones de superioridad administrativa, jurídica, financiera y de sostenibilidad ambiental. Además, los proyectos que se inscriban deben cumplir con una serie de exigencias de difícil cumplimiento para personas que toda la vida han vivido en el campo con pocas oportunidades de educarse y que poseen bajos ingresos, lo anterior indica que, históricamente, han estado en condiciones de desigualdad y exclusión.

Entre las exigencias se destacan: un esquema de viabilidad administrativa, financiera, jurídica y de sostenibilidad ambiental; un sistema que garantice la compra de la totalidad de la producción a precios de mercado; recursos manejados a través de fiducia; el estudio de títulos; la identificación de predios; y la descripción de la figura jurídica para acceder a la tierra. También se requiere de procesos asociativos; la determinación del terreno; un sistema que garantice que la tierra pueda ser adquirida por el grupo que la desea; un plan de acción para la gestión de crédito; un plan de suministro de servicios permanentes en capacitación, formación y acompañamiento; y servicios de asistencia técnica. Todo ello, con el apoyo del Ministerio de Agricultura y Desarrollo Rural, del DNP y de las entidades prestadoras del servicio de asistencia técnica directa rural, siempre y cuando los solicitantes no ostenten propiedad sobre bienes inmuebles adjudicados como baldíos después de la Ley 160 de 1994.

En este orden de ideas, los proyectos que resulten aprobados recibirán, como mínimo, los siguientes incentivos y estímulos: líneas de créditos especiales; mecanismos especiales de garantía sobre la producción; estímulos a la promoción, formación y capacitación de los campesinos, los trabajadores agrarios y las mujeres rurales; incentivos para las empresas que resulten de las alianzas concebidas bajo los requisitos de la Ley y respaldo del $100 \%$ de los recursos de los proyectos productivos a través del Fondo Agropecuario de Garantías, cuando se requiera.

Aunque la Ley promueve la incorporación de pequeños y medianos productores al proyecto como asociados para beneficiarse de la política de incentivos o estímulos, es evidente que esta asociatividad es asimétrica, 
dadas las condiciones materiales y culturales de los campesinos, las mujeres y los jóvenes rurales, incluyendo las ventajas comparativas que ostentan las empresas agroindustriales. Así las cosas, dicha incorporación puede convertirse en una práctica instrumental que incluye a los campesinos solo con el propósito de acceder a los incentivos o estímulos ofrecidos por la Ley Zidres, aprovechando dineros públicos para incentivar proyectos privados de grandes empresas.

Como bien lo dijo el senador Alberto Castilla (2015), a la vista saltan los problemas de estos proyectos asociativos:

Por un lado, promueven la vinculación de los campesinos a grandes proyectos agroindustriales en detrimento de sus posibilidades de ser propietarios o de disponer de sus tierras libremente. Aunque se contemple que los campesinos accederán a las tierras, lo harán limitadamente y ligados al megaproyecto industrial aprobado en las Zidre. Por otro, ligado a lo anterior, promueven una asociatividad en abierta desigualdad de las partes. El proyecto de ley no reconoce la asimetría existente entre los campesinos sin tierra, o con tierra y poco o nulo apoyo estatal, y los empresarios nacionales y extranjeros que, de la mano del sector financiero, de su propio capital y [ 48 ] de los numerosos incentivos y subsidios públicos de los que el campesinado carece, serán la parte fuerte de la relación y la que determine las condiciones de la asociatividad. Asimismo, las condiciones de los proyectos relacionadas con tecnología minimizan la autonomía del campesinado, desechan su conocimiento sobre la tierra y la agricultura, y buscan convertir a los campesinos en meros técnicos del campo (p. 3).

Preocupa también que los baldíos de la nación puedan ser arrendados para la agroindustria que sí tiene el capital para adecuar la tierra y para impulsar la construcción de infraestructura, lo cual no podrían hacer las familias pobres sin tierra, como lo estableció la Ley 160 de 1994. Adicionalmente, la Ley Zidres no cumplió con la consulta previa, lo cual han motivado una demanda de inconstitucionalidad que fue fallada mediante la Sentencia C-077 de 2017, en la cual la Corte Constitucional dijo, que no se requería consulta previa y que el modelo de desarrollo propuesto por la Ley no desconoce el principio de progresividad en materia de derechos sociales y derecho de asociación.

El objetivo de la Ley Zidres, que supuestamente apunta a la promoción del acceso a la propiedad para los campesinos, las mujeres y los jóvenes rurales, es engañoso, dado que la Ley 1776 de 2016 le apuesta justamente a 
lo contrario. Aunque estipule medidas que permitan que algunos campesinos detenten el título sobre sus tierras o que estipulen porcentajes sobre los cuales los campesinos asociados, eventualmente, podrán hacerse a una parte de la propiedad, la Ley tendrá como consecuencia la pérdida de tierra para los campesinos, por un lado, porque el simple título de propiedad no es garantía del pleno derecho de dominio del predio, ya que le limitan sus atributos de uso y disposición; y por el otro, al permitir la adquisición de bienes baldíos por parte de las empresas se reducen las posibilidades de adquisición a los campesinos sin tierra (Semana, 2013, junio 15; Castilla, 2015).

En síntesis, esta ley, adoptada en paralelo y por fuera del Acuerdos de Paz, contribuye a la concentración de la tierra, avala el modelo de agroindustria, reconoce a los agroempresarios como los protagonistas del campo y proyecta una imagen del campesino como incapaz de aportar al progreso económico. Dicha ley golpea los intentos de democratización de la tierra realizados por la Ley 160 de 1994; además, desconoce las sentencias de la Corte Constitucional tendientes a hacer cumplir que la destinación de los bienes baldíos en Colombia sea para quienes carecen de tierra; desconoce la obligación de la consulta previa establecida en el Convenio 169 de la Organización Internacional del Trabajo (OIT, 1989); por último, atribuye mayor importancia al derecho de acceso a la tierra sobre el derecho a la titularidad.

\subsection{Misión para la Transformación del Campo Colombiano}

Por otro lado, se encuentra la Misión para la Transformación del Campo Colombiano, también conocida como Misión Rural, iniciativa del Gobierno nacional, en cabeza del DNP en su rol de tanque de pensamiento, a través de la cual se definen los lineamientos de política pública para contar con un portafolio robusto y amplio de políticas públicas e instrumentos, con el objetivo de tomar mejores decisiones de inversión pública en el ámbito del desarrollo rural y agropecuario en los próximos veinte años que ayuden a transformar el campo colombiano. De acuerdo con José Antonio Ocampo, líder de la Misión, esta se propuso desarrollar las siguientes seis estrategias para superar las limitaciones en el campo colombiano:

- Ordenamiento y desarrollo territorial, la cual busca desarrollar instrumentos — normas, leyes, incentivos, entre otros - que regulen la propiedad, el uso, la distribución, la conservación y explotación de la tierra y los recursos naturales del territorio buscando el equilibrio entre lo social, lo económico y lo ambiental. 
- Cierre de brechas sociales con enfoque de derechos, la cual tiene como objetivo la inclusión social de los pobladores del campo a través de la provisión de bienes de interés social con modelos de intervención que respondan a las necesidades del campo.

- Inclusión productiva, cuyo objetivo es incorporar a los pobladores del campo en dinámicas económicas, complementando la estrategia de inclusión social. Se busca desarrollar mecanismos para que los pequeños productores y los trabajadores rurales se integren a los mercados, alcancen una remuneración justa y mejoren su bienestar.

- Desarrollo de una ruralidad competitiva con énfasis en el sector agropecuario, dado que el país tiene un gran potencial económico basado en la ruralidad. Esta estrategia tiene como propósito el impulso de la competitividad por medio de la provisión adecuada de bienes públicos y el fomento de un ambiente macroeconómico y financiero apropiado.

- Elementos de sostenibilidad ambiental para el desarrollo rural, con lo cual se pretende generar las condiciones de sostenibilidad para las actividades rurales por medio de instrumentos que contribuyan a la conservación del patrimonio del país en materia de aguas, suelos, biodiversidad, riqueza forestal y adaptación al cambio climático.

- Reforma institucional profunda, la cual tiene como fin contar con un arreglo institucional integral y multisectorial, con políticas claras, con amplia presencia y capacidad de ejecución en el ámbito territorial, que involucre a la sociedad civil organizada y al sector privado en la planeación, la toma de decisiones, la ejecución de programas y proyectos, y el seguimiento de resultados, con el fin de darle a los pobladores rurales un papel protagónico como gestores y actores de su propio desarrollo (Las2orillas, 2014, noviembre 12).

Sin embargo, las estadísticas del sector agropecuario (DNP, s. f.) y el Diagnóstico de la Pobreza Rural, Colombia 2010-2014 (DNP, 2015), revelan que el desarrollo rural sostenible para Colombia supone la intervención estructural del Gobierno a partir de un diagnóstico que dé cuenta de otras variables fundamentales en los territorios rurales, más allá de la pobreza. Si bien se concuerda con el diagnóstico de las desigualdades existentes en los 
territorios rurales, en los cuales se vive bajo unas profundas condiciones de pobreza y exclusión, se considera que estas variables deben posibilitar la construcción del diagnóstico a partir de una óptica plural y participativa que permita arrojar información más precisa sobre la cantidad y calidad de las necesidades básicas insatisfechas en esta población. Por otra parte, el análisis de las condiciones socioeconómicas es otra cuestión relevante, con el fin de identificar las condiciones productivas, de empleo y, en general, del aparato económico de los territorios rurales, bajo el enfoque de desarrollo territorial.

Asimismo, se precisa el análisis de los niveles de organización social y participación política de los campesinos, los jóvenes y las mujeres rurales, ya que es importante la construcción del tejido social, a efectos de dotar de sentido el territorio a través de las relaciones entre los sujetos, sus organizaciones y sus formas de participación política, entendiendo el territorio como escenario de relaciones de poder y toma de decisiones en las que la participación de los actores implicados es fundamental (Semillero de Estudios Políticos Rurales, 2010, 15 de diciembre).

\subsection{Decretos Fast Track sobre el Acuerdo de Tierras}

Recientemente fueron aprobados algunos decretos mediante el mecanismo del Fast Track, cuyo propósito principal es facilitar la implementación de la Reforma Rural Integral contemplada en el acuerdo rural de paz. Dichos decretos son los 902, 896, 893, 892, 884, 890 y 882 de 2017 , los cuales se examinan a continuación, resaltando en cada uno, los temas centrales de los que se ocupa y los asuntos problemáticos que han sido señalados por la Procuraduría General de la Nación y por la Consultoría para los Derechos Humanos y el Desplazamiento (Codhes).

\section{Luces y sombras en el proceso de implementación}

El Acuerdo de Tierras se produce en el marco de las negociaciones realizadas en La Habana y fue posible debido al cambio generado en el establecimiento en la forma de acabar con una guerra que se ha sostenido por medio siglo. Dicho cambio tuvo que ver con el reconocimiento de la existencia de un conflicto armado, el cual tiene su cimiento en las realidades socioeconómicas y políticas y que es posible superar por la vía negociada. Esto se refleja, además, en el reconocimiento del Estado a las víctimas y en su obligación de repararlas mediante la restitución de tierras (Ley 1448 de 2011). 
Tabla 1. Decretos Fast Track sobre tierras.

\begin{tabular}{|c|c|c|}
\hline $\begin{array}{c}\text { Decretos } \\
\text { Fast Track }\end{array}$ & Temas & Problemas \\
\hline $\begin{array}{l}902 \text { del } \\
29 / 05 / 17\end{array}$ & $\begin{array}{l}\text { Crea el fondo de } \\
\text { tierras para la } \\
\text { Reforma Rural } \\
\text { Integral. }\end{array}$ & $\begin{array}{l}\text { No se definea instrumentos y mecanismos para efectivamente } \\
\text { conformar y alimentar el fondo de tierras con grandes } \\
\text { latifundios inadectuadamente explotados, ni prioriza la } \\
\text { recuperación de los baldios; en cambio, crea la acción de } \\
\text { nulidad agraria con lo cual se prevéa mayores đilaciones, asi } \\
\text { como conflictos entre campesinos e indigenas en el proceso } \\
\text { para determinar los territorios ancestrales. }\end{array}$ \\
\hline $\begin{array}{l}856 \mathrm{del} \\
28 / 05 / 17\end{array}$ & $\begin{array}{l}\text { Crea el } \\
\text { Programa } \\
\text { Nacional } \\
\text { Integral de } \\
\text { Sustitución de } \\
\text { Cultivos de Uso } \\
\text { Ilicito (PNIS). }\end{array}$ & $\begin{array}{l}\text { Tener claras las fuentes de tierras implica la reversión de los } \\
\text { baldios adjudicados fraudulentamerte. Se requiere una revisión } \\
\text { de los titulos mineros para derle prioridad a las comunidades } \\
\text { campesinas y étricas; que victimas, las comunidades étnicas y } \\
\text { los campesinos gue no han tenido acceso a la tierra o no gozan } \\
\text { de su titularidad sean realmente los sujetos de derechos de la } \\
\text { reforma: y que simultáneamente estos territorios sean reparados } \\
\text { de manera colectiva. }\end{array}$ \\
\hline $\begin{array}{l}893 \text { del } \\
28 / 05 / 17\end{array}$ & $\begin{array}{l}\text { Crea los } \\
\text { Programas de } \\
\text { Desarrollo con } \\
\text { Enfoque } \\
\text { Territorial } \\
\text { (PDET). }\end{array}$ & $\begin{array}{l}\text { Los aspectos cuestionados a este decreto, seguin Codbes (2017), } \\
\text { son la autonomia territorial, la consulta previa, libre e } \\
\text { informada, y el derecho a la reparación de las victimas. }\end{array}$ \\
\hline $\begin{array}{l}892 \mathrm{del} \\
28 / 05 / 17\end{array}$ & $\begin{array}{l}\text { Crea un régimen } \\
\text { transitorio para } \\
\text { la acreditación } \\
\text { en alta calidad } \\
\text { de los } \\
\text { programas } \\
\text { acadtmicos de } \\
\text { licenciatura } \\
\text { (PAL) en } \\
\text { pregrados. }\end{array}$ & $\begin{array}{l}\text { Flexibilización de requisitos exigidos por el Ministerio de } \\
\text { Educación, vulneración del derecho a la igualdad versus medida } \\
\text { razonable en zonas rurales o afectadas por el conflicto y por la } \\
\text { pobreza. }\end{array}$ \\
\hline $\begin{array}{l}884 \mathrm{del} \\
26 / 05 / 17\end{array}$ & $\begin{array}{l}\text { Normas } \\
\text { tendientes a } \\
\text { implementación } \\
\text { del Plan } \\
\text { Nacional de } \\
\text { Electrificación } \\
\text { Rural ea el } \\
\text { marco del } \\
\text { Acuerdo FinaL. }\end{array}$ & $\begin{array}{l}\text { En general, el Decreto responde lo que senala el Acto } \\
\text { Legislativo para la Paz, sin embargo, se resaltan dos elementos } \\
\text { ausentes en el Decreto: a) no se hace mención especifica a la } \\
\text { instalación do infraestructura necesaria para el servicio de } \\
\text { energia electrica; b) no se hace mención al acceso comanitario a } \\
\text { Internet. } \\
\text { Además, el Ministerio de Minas y Energin es el respoasable de } \\
\text { la implementación de los Planes sin veeduria local o de alguna } \\
\text { otra entidad. Esto podria suponer presicnes en relación con } \\
\text { consultas populares relacionadas coo la explotación minero- } \\
\text { energética; por ello es necesario definir contrapesos en este } \\
\text { proceso o establecer veedurias ciudadanas. }\end{array}$ \\
\hline $\begin{array}{l}890 \text { del } \\
28 / 05 / 17\end{array}$ & $\begin{array}{l}\text { Formulación del } \\
\text { Plan Nacional } \\
\text { de Construcción } \\
\text { y Mejoramieato } \\
\text { de Vivierda } \\
\text { Social Rural. }\end{array}$ & $\begin{array}{l}\text { El Decreto utiliza verbos no programáticos como epromoción: } \\
\text { o esocializacións, sia un lenguaje de garantia de derechos. Esto } \\
\text { ocurre, de manera particular, en lo que se refiere al punto de } \\
\text { soluciones tecnológicas para el acceso a agea potable y } \\
\text { trataniento de aguas residusles (Codhes, } 2017 \text { ), ademís, los } \\
\text { subsidios de vivienda de interés social rural serán operados por } \\
\text { ua actor privado, lo cual va en contravia de la implementación } \\
\text { de los acuerdos con enfoque territorial, lo cual supone } \\
\text { fortalecimiento de las autoridades locales y participación de la } \\
\text { comunidad. }\end{array}$ \\
\hline $\begin{array}{l}882 \text { del } \\
26 / 05 / 17\end{array}$ & $\begin{array}{l}\text { Normas sobre } \\
\text { servicio } \\
\text { educativo estatal } \\
\text { y profesion } \\
\text { docente. }\end{array}$ & $\begin{array}{l}\text { Para Codhes es va decreto ley operativo que describe el proceso } \\
\text { mediante el cual se seleccionara la planta educativa en los } \\
\text { municipios priorizados para la implementación de PDET. No } \\
\text { incorpora medidas o mecanismos de accesa, ni hace refereocia a } \\
\text { procesos inclusivos o de etnoedacación. De hecho, señala que } \\
\text { todo será reglamentado por circulares del Ministerio de } \\
\text { Educación Nacicnal. } \\
\text { Por su parte, la Procuraduria General de la Nación considera } \\
\text { que son medidas necesarias que se encueatran acordes con la } \\
\text { Constitución Politica para garantizar el derecho fundamental a } \\
\text { la educación en zonas que han estado afectadas por la violencia. }\end{array}$ \\
\hline
\end{tabular}

Fuente: elaboración propia a partir de Procuraduría (2017) y Codhes (2017). 
La reivindicación fundamental de las FARC-EP, con la cual justifican su alzamiento y su programa, es la pobreza del campesinado y la ausencia de una reforma agraria en un país donde la concentración de la tierra, el despojo de millones de hectáreas de tierras de los campesinos por la violencia y el abandono del Estado han sido las causas de la miseria de millones de campesinos, comunidades indígenas y comunidades afrodescendientes. De allí que el reconocimiento de esa realidad en el Acuerdo, la disposición de un fondo de tierras de tres millones de hectáreas para distribuir gratuitamente entre campesinos pobres, la formalización de la propiedad sobre la tierra de siete millones de hectáreas y la disposición de planes nacionales para proveer de bienes públicos a las zonas campesinas constituyan un significativo avance en el concepto de «bienestar y buen vivir» que recoge repetidamente el texto del Acuerdo.

Si se le suma el objetivo de la Ley de Víctimas y Restitución de Tierras (Ley 1448 de 2011) se crea una expectativa muy significativa para la verdadera transformación del campo, lo que el Acuerdo denomina Reforma Rural Integral. Sin embargo, hay que recordar que el Gobierno nacional repetidamente afirmó que en La Habana no se estaba negociando ni el modelo de desarrollo, ni el modelo militar del país, lo que significa que tampoco el modelo de desarrollo agrario del gobierno Santos, que está atado a la gran producción agroindustrial, a los tratados de libre comercio para la exportación y al beneficio de las empresas y empresarios privados del campo, está comprometido con el Acuerdo de Paz en materia de tierras.

Incluso esta «locomotora» del modelo de desarrollo de Santos y la «locomotora minera» están exentas de discusión y tratamiento en el Acuerdo de Tierras de La Habana. Esto explica por qué, por ejemplo, ya publicado desde mayo de 2013 el Acuerdo de Tierras, el Gobierno expidió a principios de 2016 la Ley Zidres, la cual intenta legalizar la apropiación ilegal de baldíos de la nación en manos del gran empresariado agroindustrial nacional y transnacional, y explica también cómo tras la derrota del plebiscito en octubre de 2016, en la renegociación que estableció con los sectores del No, el Gobierno pretendiera demostrar que no se estaba poniendo en entredicho la propiedad privada, no se estaba negociando un nuevo modelo económico, ni el Estado de derecho, ni se estaba comprometiendo la seguridad jurídica de las grandes empresas agropecuarias. Dicha renegociación, en cambio, puso en entredicho el acento en el carácter redistributivo de la propiedad de la tierra y la necesidad de catastro rural en beneficio del pequeño campesino y del desarrollo territorial. 
Ahora bien, en lo que tiene que ver con los decretos del Fast Track, resulta preocupante la gran burocratización y recentralización en contra de lo que el mismo acuerdo llama paz territorial, donde la participación de las regiones parece reducida a aplicar y ejecutar las orientaciones que desde el Gobierno central se definan, lo cual va en contravía del principio de autonomía territorial previsto en el artículo 287 de la Constitución Política, del cual se desprende la descentralización y los principios de coordinación, concurrencia y subsidiariedad. A esto se suma la falta de la consulta previa e informada a las comunidades rurales en temas que son trascendentales para su desarrollo y una ausencia de criterios de diferenciación entre los planes de reparación a las víctimas del conflicto (Ley 1448 de 2011) y las obligaciones derivadas de la política social del Estado.

\section{Retos. A manera de conclusión}

Todos los anteriores problemas identificados, a través de un diagnóstico socioeconómico del campo colombiano, revelan que son muchas las necesidades del campo y muchos los retos que enfrenta el Gobierno nacional para garantizar un desarrollo rural integral, como lo prometió en el primer acuerdo de La Habana. Uno de ellos, y quizá el más importante,

[ 54 ] es la reconfiguración de un nuevo modelo de desarrollo distinto al modelo neoliberal y distinto al modelo propuesto en la Ley Zidres, el cual favorece a los empresarios agroindustriales, en menoscabo de los intereses de los campesinos, los jóvenes y las mujeres rurales. Ese nuevo modelo de desarrollo precisa de unos enfoques centrados en los actores del campo y en sus necesidades, para lo cual podrían ser útiles el enfoque de derechos, el enfoque territorial y el enfoque diferencial por etnia, raza y género.

Se requiere también repensar la ocupación y el uso del territorio a partir de un nuevo reordenamiento del mismo, dado que en la actualidad peligran zonas de reserva forestal, se realizan actividades económicas y productivas con predominio de la ganadería y la minería sobre la agricultura, proliferan y siguen creciendo las actividades económicas ilegales sin que se haya planteado una solución de fondo, sigue avanzando el proceso de colonización y ampliación de la frontera agrícola en la Orinoquía y la Amazonía. Por otra parte, el cambio climático continúa provocando tragedias invernales y se precisa de una efectiva gestión del riesgo, así como de una delimitación de la frontera agropecuaria, lo cual sigue siendo una tarea urgente. 
Otro aspecto fundamental en la propuesta de Desarrollo Rural Integral es la Tenencia de la tierra para el desarrollo humano. La tierra es un elemento estratégico para alcanzar el desarrollo humano, de allí la importancia de abordar problemas históricos de gran envergadura como el conflicto rural y la tierra, la estructura de la tenencia de la tierra, la estructura de tamaños de la propiedad, la propiedad y la tenencia femenina de tierras. Todos estos con enfoques de desarrollo humano y de equidad.

Con este panorama, lo que se espera es que los acuerdos generados en La Habana contribuyan a resolver los problemas de la violencia y la persistencia del orden social rural por el desconocimiento político del campesinado, además de la violencia contra la población rural a través de las disputas por el territorio, el desplazamiento forzado y el despojo de tierras; así como la intervención de grupos armados en la política, la existencia de élites y sus prácticas clientelares y corruptas, entre otras. En síntesis:

Colombia es más rural de lo que se cree, pero cuenta hoy con más hectáreas en minería que en producción de alimentos. El Gobierno firma tratados y asociaciones de libre comercio y crea incentivos para el empresariado agroindustrial, pero, con honrosas excepciones, el desempeño productivo agropecuario deja mucho que desear. Entre tanto, sectores de pequeños y medianos campesinos esperan del Estado medidas de más envergadura para evitar que sus economías desaparezcan o queden reducidas apenas a medios de sobrevivencia (PNUD, 2011).

Esto requiere, como se ha dicho antes, de cambios estructurales en la esfera social rural, ya que los efectos del conflicto han sido múltiples, profundos, y su reparación y no repetición precisan de la voluntad política, el cambio de enfoques, un nuevo modelo económico, más institucionalidad, mayor presupuesto, un gran ánimo y la decisión de reconciliación.

\section{Referencias bibliográficas}

1. Asamblea Nacional Constituyente, República de Colombia. (1991). Constitución Política de Colombia. Bogotá, D. C.: Legis.

2. Bedoya Bedoya, María Rocío. (2015, 22-24 de septiembre). Política minera con enfoque territorial, el caso de Marmato en Colombia. Tercer Seminario Internacional: Territorios, sustentabilidad, movimientos sociales y ciudadanía. Escuela Superior de Administración Pública (ESAP), Popayán. 
3. Castilla, Alberto. (2015). La disputa por los baldíos: ¿el campo para los inversionistas o para el campesinado? Semanario Virtual Caja de Herramientas, 475. Recuperado de http://viva.org.co/cajavirtual/svc0475/articulo01.html

4. Centro Nacional de Memoria Histórica (CNMH). (2013). ¡Basta ya! Colombia: memorias de guerra y dignidad. Bogotá, D. C.: CNMH.

5. Consultoría para los Derechos Humanos y el Desplazamiento Forzado (Codhes). (2017). Comentarios generales a decretos Fast Track que tienen impacto en los derechos de las víctimas. Recuperado de http://www.codhes.org/ codhes/ images/CODHEScomentariosdecretosleyfasttrackvictimas_final.pdf

6. Colombia. Congreso de la República. Ley 200. (30 de diciembre de 1936). Sobre régimen de tierras. Diario Oficial. Bogotá, D. C., 1937. N. ${ }^{\circ} 23388$. p. 3.

7. Colombia. Congreso de la República. Ley 100. (31 de diciembre de 1944). Ley de aparcerías. Diario Oficial. Bogotá, D. C., 1945. N. ${ }^{\circ} 25759$. p. 2.

8. Colombia. Congreso de la República. Ley 135. (15 de diciembre de 1961). Sobre reforma social agraria. Diario Oficial. Bogotá, D. C., 1961. N. 30691. p. 801.

9. Colombia. Congreso de la República. Ley 1. (26 de enero de 1968). Por la cual se introducen modificaciones a la Ley 135 de 1961 sobre reforma social agraria. Diario Oficial. Bogotá, D. C., 1968. N. 32428. p. 1.

10. Colombia. Congreso de la República. Ley 4. (29 de marzo de 1973). Por la cual se introducen modificaciones a las Leyes 200 de 1936, 135 de 1961 y $1^{\text {a }}$ de 1968. Diario Oficial. Bogotá, D. C., 1973. N. 33828. p. 145.

[ 56 ] 11. Colombia. Congreso de la República. Ley 6. (10 de enero de 1975). Por la cual se dictan normas sobre contratos de aparcería y otras formas de explotación de la tierra. Diario Oficial. Bogotá, D. C., 1968. N. 34244. p. 190.

12. Colombia. Congreso de la República. Ley 160. (3 de agosto de 1994). Por la cual se crea el Sistema Nacional de Reforma Agraria y Desarrollo Rural Campesino. Diario Oficial. Bogotá, D.C, 1994. N. ${ }^{\circ} 41479$.

13. Colombia. Congreso de la República. Ley 1448. (10 de junio de 2011). Por la cual se dictan medidas de atención, asistencia y reparación integral a las víctimas del conflicto armado interno y se dictan otras disposiciones. Recuperado de https://www. unidadvictimas.gov.co/sites/default/files/documentosbiblioteca/ley-1448-de-2011.pdf

14. Colombia. Congreso de la República. Ley 1776. (29 de enero de 2016). Ley Zidres por la cual se crean y se desarrollan las zonas de interés de desarrollo rural, económico y social. Diario Oficial. Bogotá, D. C., 2016. N. ํ 49770.

15. Colombia. Corte Constitucional. Sentencia C-077. (8 de febrero de 2017). Recuperado de http://www.corteconstitucional.gov.co/relatoria/2017/C-077-17.htm

16. Colombia. Departamento Nacional de Planeación (DNP). Documento Conpes 3917. (7 de febrero de 2018). Áreas de referencia como insumo para la identificación de las zonas de interés de desarrollo rural, económico y social (Zidres). Recuperado de https://colaboracion.dnp.gov.co/CDT/Conpes/Econ\%C3\%B3micos/3917.pdf 
17. Colombia. Presidencia de la República. Decreto Ley 1273. (3 de agosto de 2016). Por el cual se adiciona una Parte al Libro 2 del Decreto 1071 de 2015, Decreto Único Reglamentario del Sector Administrativo Agropecuario, Pesquero y de Desarrollo Rural relacionada con las Zonas de Interés de Desarrollo Rural, Económico y Social (Zidres). Diario Oficial. Bogotá, D. C., 2016. N. 49954. p. 1.

18. Colombia. Presidencia de la República. Decreto Ley 882. (26 de mayo de 2017). Por el cual se adoptan normas sobre la organización y prestación del servicio educativo estatal y el ejercicio de la profesión docente en zonas afectadas por el conflicto armado. Recuperado de http://es.presidencia.gov.co/normativa/normativa/ DECRETO\%20882\%20DEL\%2026\%20DE\%20MAYO\%20DE\% 202017.pdf

19. Colombia. Presidencia de la República. Decreto Ley 884. (26 de mayo de 2017). Por el cual se expiden normas tendientes a la implementación del Plan Nacional de Electrificación Rural en el marco del Acuerdo Final para la Terminación del Conflicto y la Construcción de una Paz Estable y Duradera. Recuperado de http:// es.presidencia.gov.co/normativa/normativa/DECRETO $\% 20884 \% 20$ DEL $\% 2026 \% 20$ DE\%20MAYO\%20DE\%202017.pdf

20. Colombia. Presidencia de la República. Decreto Ley 890. (28 de mayo de 2017). Por el cual se dictan disposiciones para la formulación del Plan Nacional de Construcción y Mejoramiento de Vivienda Social Rural. Recuperado de http:// es.presidencia.gov.co/normativa/normativa/DECRETO\%20890\%20DEL\%2028\%20 DE\%20MAYO\%20DE\%202017.pdf

21. Colombia. Presidencia de la República. Decreto Ley 892. (28 de mayo de 2017). Por el cual se crea un régimen transitorio para la acreditación en alta calidad de los programas académicos de licenciaturas a nivel de pregrado que son ofrecidos en departamentos donde se localizan municipios priorizados para la implementación de los Programas de Desarrollo con Enfoque Territorial (PDET). Recuperado de http:// es.presidencia.gov.co/normativa/normativa/DECRETO\%20892\%20DEL\%2028\%20 DE\%20MAYO\%20DE\%202017.pdf

22. Colombia. Presidencia de la República. Decreto Ley 893. (28 de mayo de 2017). Por el cual se crean los Programas de Desarrollo con Enfoque Territorial (PDETR). Recuperado de http://es.presidencia.gov. co/normativa/normativa/DECRETO $\% 20$ 893\%20DEL\%20 28\%20DE\%20MAYO\%20DE\%202017.pdf

23. Colombia. Presidencia de la República. Decreto Ley 902. (29 de mayo de 2017). Por el cual se adoptan medidas para facilitar la implementación de la Reforma Rural Integral contemplada en el Acuerdo Final en materia de tierras, específicamente el procedimiento para el acceso y formalización y el Fondo de Tierras. Recuperado de http://es.presidencia.gov.co/normativa/normativa/DECRETO \%20902\%20DEL \% 20 29\%20DE\%20MAYO\%20DE\%202017.pdf

24. Colombia. Presidencia de la República. Decreto Ley 896. (29 de mayo de 2017). Por el cual se crea el Programa Nacional Integral de Sustitución de cultivos de uso ilícito (PNIS). Diario oficial. Bogotá, D. C., 2017. N. ${ }^{\circ} 50248$. 
25. Departamento Nacional de Planeación (DNP). (2015). Diagnóstico de la pobreza rural. Colombia 2010-2014. Recuperado de https://colaboracion.dnp.gov. co/CDT/Agriculturapecuarioforestal \%20y\%20pesca/Pobreza\%20Rural.pdf

26. Departamento Nacional de Planeación (DNP). (s. f.). Estadísticas del sector agropecuario. Recuperado de https:/www.dnp.gov.co/programas/agricultura/ estadisticas-del-sector-agropecuario/Paginas/estadisticas-sector-agropecuario.aspx

27. Fajardo, Darío. (1984). Haciendas, campesinos y políticas agrarias en Colombia, 1920-1980. Bogotá, D. C.: Oveja Negra.

28. Gobierno de Colombia y FARC-EP. (2017). Acuerdo final para la terminación del conflicto y la construcción de una paz estable y duradera. Bogotá, D. C.: Desde Abajo.

29. Las2Orillas. (2014, noviembre 12). ¿Qué propone la misión rural para el campo colombiano? Recuperado de http://www.las2orillas.co/que-propone-la-misionrural-para-el-campo-colombiano/

30. Organización Internacional del Trabajo (OIT). (1989). C-169 convenio sobre pueblos indígenas y tribales. Recuperado de https://www.ilo.org/dyn/normlex/es/f?p $=$ NORMLEXPUB:12100:0::NO::P12100_INSTRUMENT_ID:312314

31. Palacios, Marco. (1995). Entre la legitimidad y la violencia. Colombia 18751994. Bogotá, D. C.: Norma.

32.ProcuraduríaGeneraldelaNacióndeColombia.(2017).Conceptosdel procurador. Recuperado de https://www.procuraduria.gov.co/portal/index.jsp?option = co.gov.pgn.

[ 58 ] portal.frontend.component.pagefactory.ConceptoProcuComponentPageFactory

33. Programa de las Naciones Unidas para el Desarrollo (PNUD). (2011). Colombia rural. Razones para la esperanza. Informe Nacional de Desarrollo Humano 2011. Recuperado de http://escuelapnud.org/biblioteca/documentos/abiertos/06 indh2011co.pdf

34. Semillero de Estudios Políticos Rurales UdeA. (2010, 15 de diciembre). Diez tesis sobre el problema de la tierra en Colombia. Foro Tierra una condición para el cambio, Universidad de Antioquia, Medellín.

35. Semana. (2013, junio 15). El chicharrón de los baldíos. Recuperado de http:// www.semana.com/nacion/articulo/el-chicharron-baldios/346489-3

36. Vargas Velásquez, Alejo. (2013). Balance después de Oslo: ruidos y desencantos, pero todo sigue adelante. Semanario Virtual Caja de Herramientas, 326. Recuperado de http:/viva.org.co/cajavirtual/svc0326/articulo04.html

37. Villamizar Herrera, Darío. (2017). Las guerrillas en Colombia. Una historia desde los orígenes hasta los confines. Bogotá, D. C.: Géminis.

38. Villarraga Sarmiento, A. (2016). Balance de la negociación de paz GobiernoFARC. Semanario Virtual Caja de Herramientas, 479. Recuperado de http://viva.org. co/cajavirtual/svc0479/articulo03.html 